This item was submitted to Loughborough's Research Repository by the author.

Items in Figshare are protected by copyright, with all rights reserved, unless otherwise indicated.

\title{
Metacognitive and metamemory beliefs in the development and maintenance of posttraumatic stress disorder
}

PLEASE CITE THE PUBLISHED VERSION

http://dx.doi.org/10.1177/2167702616649348

\section{PUBLISHER}

Association for Psychological Science / Sage

VERSION

AM (Accepted Manuscript)

\section{PUBLISHER STATEMENT}

This work is made available according to the conditions of the Creative Commons Attribution-NonCommercialNoDerivatives 4.0 International (CC BY-NC-ND 4.0) licence. Full details of this licence are available at: https://creativecommons.org/licenses/by-nc-nd/4.0/

\section{LICENCE}

CC BY-NC-ND 4.0

\section{REPOSITORY RECORD}

Takarangi, Melanie K., Rashelle A. Smith, Deryn Strange, and Heather Flowe. 2016. "Metacognitive and Metamemory Beliefs in the Development and Maintenance of Posttraumatic Stress Disorder". Loughborough University. https://hdl.handle.net/2134/23533. 
Metacognitive and Meta-Memory Beliefs in the Development and Maintenance of

Posttraumatic Stress Disorder

\author{
Melanie K. T. Takarangi ${ }^{1}$ \\ Rashelle A. Smith ${ }^{1}$ \\ Deryn Strange ${ }^{2}$ \\ $\&$ \\ Heather D. Flowe ${ }^{3}$
}

\begin{abstract}
${ }^{1}$ School of Psychology, Flinders University
${ }^{2}$ Department of Psychology, John Jay College of Criminal Justice, CUNY

${ }^{3}$ School of Sport, Exercise, and Health Sciences, Loughborough University
\end{abstract}

Corresponding Author:

Melanie Takarangi

School of Psychology

Flinders University

GPO Box 2100

Adelaide

South Australia, 5001

melanie.takarangi@flinders.edu.au 


\begin{abstract}
Can metacognition increase trauma sufferers' risk for developing and maintaining posttraumatic stress disorder (PTSD)? We assessed the role of a range of cognitive and metacognitive belief domains — including meta-memory — in PTSD symptoms. Adult participants reported their existing meta/cognitions and lifetime exposure to trauma, then twelve weeks later, they reported meta/cognitions and PTSD symptoms in relation to new trauma exposure since the initial assessment. Participants with more PTSD symptoms held more problematic metacognitions than participants with fewer distress symptoms. Moreover, people who endorsed maladaptive metacognitions before trauma exposure were more likely to experience symptoms of PTSD after exposure. Metacognition predicted the maintenance of elevated PTSD symptoms over the twelve-week delay. Our findings support the metacognitive model of PTSD and highlight the importance of meta-memory, an understudied factor in PTSD research.
\end{abstract}


Metacognitive and Meta-Memory Beliefs in the Development and Maintenance of Posttraumatic Stress Disorder

Exposure to a sudden or sustained stressful experience can lead to psychological problems such as posttraumatic stress disorder (PTSD). Hallmark symptoms of PTSD include repeated and unwanted re-experiencing of the event, negative alterations in arousal, reactivity, cognition and mood, and active avoidance of trauma reminders (American Psychiatric Association, 2013). Yet, attesting to human resilience, not all trauma-exposed people develop PTSD (Lee, 2006). Determining why some trauma-exposed people develop serious psychopathology when others do not is of critical clinical significance. Recently, metacognition— beliefs about thinking that guide our thinking and coping — has received attention for its role in PTSD (Wells, 2000). PTSD sufferers who endorse maladaptive metacognitive beliefs post-trauma tend to exhibit more PTSD symptoms (e.g., Roussis \& Wells, 2006). However, research to date has not examined the role metacognition might play in trauma reactions over time. Here, we examined whether dysfunctional metacognition pretrauma predicted PTSD symptomatology post-trauma, and whether metacognitive beliefs predicted the maintenance of elevated PTSD symptom levels over time.

Wells' (2000; Wells \& Sembi, 2004) metacognitive model focuses on how people's metacognitive beliefs can lead to PTSD. It stipulates that intrusions, startle responses and increased arousal are normal responses to trauma, forming part of a self-righting, reflexive adaptation process (RAP) that initiates automatically and determines adjustment and recovery. The RAP's goal is to simulate plans for future threats. Thus, symptoms should subside once a satisfactory plan is established. However, metacognitive beliefs that encourage dysfunctional thinking styles or maintain focus on danger or the person's unsatisfactory reactions to trauma-worry/rumination, thought suppression, threat 
monitoring — can obstruct the RAP and thus interfere with spontaneous recovery from trauma (Wells \& Sembi, 2004).

Researchers have investigated a range of maladaptive cognitive and metacognitive beliefs trauma-exposed people hold. We know people who negatively appraise their traumatic experience are at increased risk of pathology (Foa, Ehlers, Clark, Tolin, \& Orsillo, 1999) and people who interpret their intrusive memories negatively are less able to overcome their posttraumatic symptoms (e.g., Ehlers, Mayou, \& Bryant, 1998; Halligan, Michael, Clark, \& Ehlers, 2003). Recent evidence suggests that training people to adopt a positive appraisal style regarding their ability to appropriately respond to trauma led to fewer analogue symptoms (Woud, Holmes, Postma, Dalgleish \& Mackintosh, 2012). Moreover, Kleim et al. (2013) found that changes in dysfunctional trauma-related appraisals led to decreased PTSD symptoms among PTSD patients who received trauma-focused cognitive behavior therapy.

Indeed, people who hold maladaptive beliefs pre-trauma may be predisposed to develop PTSD. Bryant and Guthrie (2005) found that trainee firefighters with a pre-existing tendency for negative self-appraisal—but not cognition concerning self-blame or the world as unsafe-were more symptomatic six months later. These results suggest that studying people's cognition - and perhaps their metacognition — pre-trauma may help identify people most at risk for PTSD symptomatology.

Indeed, Bennett and Wells (2010) found student nurses who endorsed negative metamemory beliefs (e.g., "having gaps in memory of the event means I am not normal") were more likely to exhibit PTSD symptoms after a distressing event during their training. Moreover, such beliefs predicted PTSD better than objective indicators of memory problems in participants' recall narratives. These data suggest that metacognitive beliefs about memorial problems may be an important area for further research. 
In summary, extant research demonstrates that dysfunctional meta/cognition may render people more vulnerable to increased PTSD symptomatology. Does metacognition also play a role in maintaining posttraumatic stress? Some studies show that post-trauma cognition independently predicts PTSD when measured between several months and one-year post trauma (e.g., Ehlers et al., 1998; Halligan et al., 2003), and, in children, mediates the relationship between initial and longer-term PTSD symptoms (Meiser-Stedman, Dalgleish, Glucksman, Yule \& Smith, 2009). However, to our knowledge, no study has investigated whether metacognition contributes to the maintenance of persistent PTSD symptoms in adults over time.

\section{The Present Study}

We investigated the role of cognitive and metacognitive factors in predicting and maintaining PTSD among a non-clinical adult population. Even short research timeframes can capture a high prevalence of trauma in non-clinical populations (e.g., eight weeks; Frazier et al., 2009). However, to increase the likelihood that we would capture trauma, we assessed participants over twelve-weeks. We measured participants' trauma-related cognition, metacognition and PTSD symptoms pre- and post- any recent trauma exposure. Our aims were threefold. First, we examined the cross-sectional relationship between a range of cognitive and metacognitive belief domains — including positive and negative meta-memory beliefs - and PTSD reactions to traumatic events. Second, we examined whether pre-existing metacognitive beliefs (T1) increased PTSD symptomatology after trauma (T2). Third, we investigated whether metacognition predicted the maintenance of elevated PTSD symptom levels over time. 


\section{Method}

\section{Participants}

Participants $(N=664)$ were residents of Australia $(n=74)$, Canada $(n=17)$, New Zealand ( $n=4)$, the UK $(n=51)$ and USA $(n=518)$, at least 18 years old, and fluent in English. We recruited participants from Amazon Mechanical Turk ( $n=372$; these participants received $\$ 0.75$ at Time 1 and $\$ 0.75$ at Time 2, in keeping with rates of compensation by time for other psychology studies), the Flinders University Psychology Research Participation Pool ( $n=33$, course credit), and standard research recruitment websites and social networking sites ( $n=259$, voluntary participation). Of the 683 who completed the Time 1 (T1) survey, 315 also participated at Time 2 (T2). We embedded manipulation checks to ensure participants paid attention and excluded participants who failed more than one ( $n=13$ at T1, $n=10$ at T2). From T1, we also excluded five participants who did not meet the eligibility criteria, and one participant who completed the survey twice. From T2, we were unable to use one participant who had failed attention checks at T1, one participant who could not be matched to their T1 data, and three participants who completed T2 18 weeks or more after T1. The final sample comprised $664 \mathrm{~T} 1$ participants and $300 \mathrm{~T} 2$ participants. Table 1 shows their demographics and descriptive information for the main measures. Notably, at T1, participants who did not complete T2 were significantly older $(p<.01)$, reported significantly more PTSD symptoms $(p<.01)$, and significantly higher problematic cognition and metacognition on all but positive meta-memory beliefs $(p s<.05)$, compared to completers.

This research was approved by the Social and Behavioural Research Ethics Committee at Flinders University and conducted in accordance with the provisions of the World Medical Association Declaration of Helsinki.

\section{Measures}

We administered all measures at T1 and T2, twelve weeks apart. 
Personal information. Participants completed demographic questions (age, ethnicity, country of residence, highest education level achieved) and provided their email address.

Depression and anxiety. The 14-item Hospital Anxiety and Depression Scale (HADS, Zigmund \& Snaith 1983) assessed anxiety and depression symptoms. Participants rated each item from 0-3 according to how they felt during the previous week (anchors vary by item). The depression subscale focuses mainly on the reduced pleasure response aspect of depression (e.g., "I feel cheerful"; $0=$ Most of the time, $3=$ Not at all) while the anxiety subscale focuses on generalized anxiety and panic (e.g., "worrying thoughts go through my mind"; $0=$ Only occasionally, $3=\mathrm{A}$ great deal of the time). For our sample, internal consistency was: Anxiety: $\mathrm{T} 1=.81, \mathrm{~T} 2=.88$; Depression: $\mathrm{T} 1=.73, \mathrm{~T} 2=.87$.

Traumatic events. The Trauma History Screen (THS; Carlson et al., 2011) assessed lifetime exposure to potentially traumatic events at T1; at T2, participants indicated whether they had experienced any of the events since T1. Participants also specified their age (or days since the event for T2), and how emotionally distressed they were at the time of the event (not at all/a little/somewhat/much/very much). Next, participants indicated their selfnominated worst event and described that event briefly. The psychometric properties of this scale are comparable or better than longer measures of trauma exposure (Carlson et al., 2011).

Posttraumatic stress symptoms. The PTSD Checklist-Specific Version (PCL; Weathers, Litz, Herman, Huska, \& Keane, 1993) assessed posttraumatic stress. Participants responded to 17 items regarding their self-nominated worst event at T1 and T2 and their symptoms within the past two weeks, using a 5 -point scale ( $1=$ not at all $-5=$ extremely). The internal consistency in the current sample was $\mathrm{T} 1=.95$ and $\mathrm{T} 2=.95$. To investigate symptom maintenance over the T1-T2 delay, we classified participants' PTSD symptoms as 'persistent' if they displayed elevated PCL scores ( $>44)$ at T1 and T2, and as 'recovered' if they 
displayed elevated scores at T1 but not at T2 (that is, we followed: Blanchard, JonesAlexander, Buckley, \& Foreris, 1996; Bonanno, 2005; Robinaugh et al., 2011).

Trauma related cognitions. The Posttraumatic Cognitions Inventory (PTCI; Foa et al., 1999) measured negative cognitions about the self (e.g., "I can't rely on myself"), and the world (e.g., "you can never know what or who may harm you"), and self-blame (e.g., "the event happened because of the way I acted"). Participants rated their agreement with each statement $(1=$ totally disagree $-7=$ totally agree $)$. Here, the PTCI demonstrated adequate reliability for each subscale (self: .96 \& .97; world: .92 \& .91; self-blame: .88 \& .94).

Metacognitive beliefs. We selected three subscales of the Metacognitions Questionnaire-30 (MCQ-30; Wells \& Cartwright-Hatton, 2004) due to their established association with PTSD (Roussis \& Wells, 2006): positive beliefs about worry (e.g. "worrying helps me cope"), beliefs about the uncontrollability and danger of thoughts (e.g. "my worrying is dangerous for me"), and beliefs about the need to control thoughts (e.g. "it is bad to think certain thoughts"). Participants rated their agreement with each statement on a 4point scale ( $1=$ do not agree $-4=$ agree very much). The MCQ-30 is well validated in adults (Wells \& Cartwright-Hatton, 2004). Cronbach's alpha ranged from .77 to .92. The 10-item Response to Intrusions Questionnaire (RIQ; Clohessy \& Ehlers, 1999) ${ }^{1}$ measured negative inferences about the meaning of intrusive memories. Participants who had experienced intrusions rated what they thought their intrusions meant (e.g., "my life is ruined") on a 7point scale ( $1=$ totally disagree $-7=$ totally agree). The scale has previously shown good reliability and predictive validity (e.g., Clohessy \& Ehlers, 1999; Halligan et al., 2003). Here, internal consistency was $\mathrm{T} 1=.92$ and $\mathrm{T} 2=.91$.

Meta-memory. The Beliefs about Memory Questionnaire (BAMQ; Bennett \& Wells, 2010) assessed metacognition about traumatic memory. The 15 items measured positive

\footnotetext{
${ }^{1}$ Due to an initial coding error on the survey, we were unable to use T1 RIQ data for 92 participants.
} 
beliefs about needing a complete trauma memory (e.g., "I must try to remember all of the details of the event so that I can understand why it happened") and negative beliefs about the consequences of not having a complete memory (e.g., "gaps in my memory for the event are preventing me from getting over it"). Participants rated their agreement with each belief on a 4-point Likert scale (1=Do not agree-4=Agree very much). Bennett and Wells (2010) found preliminary support for the convergent validity of the BAMQ subscales with subscales of the MCQ-30. Here, the internal consistency was .91 (T1) and .92 (T2) for positive beliefs and .85 for negative beliefs at both $\mathrm{T} 1$ and $\mathrm{T} 2$.

Because responses on the PTCI, BAMQ and RIQ are anchored to a specific traumatic event, we asked participants to respond in reference to their worst nominated event or, if they had not described one, a negative event that they had experienced.

Procedure

At T1, participants completed the questionnaire battery online. Approximately 12 weeks later, we emailed participants who consented to be recontacted with the link to the T2 survey (completion time $=77-91$ days after T1). Participants received full debriefing information at the study conclusion.

\section{Results}

All analyses were two-tailed and alpha was set at .05. Several subscale scores at T1 and T2 were positively skewed. Although square root and log transformations reduced the skew, the overall pattern of results was the same; thus, we retained the original untransformed data for analysis. We replaced missing items on questionnaires using mean substitution (by subscale). 
Consistent with prior studies, most ( $n=633,95.3 \%)$ participants reported experiencing at least one lifetime trauma (Breslau et al., 1998; Frazier et al., 2009). ${ }^{2}$ The most frequent self-nominated worst events were: unexpected death of a loved one $(n=173)$, “other" trauma ( $n=105$, e.g., childhood emotional abuse, stillbirths, kidnappings), child sexual assault $(n=67)$, sudden abandonment $(n=48)$ and transport accident $(n=46)$. At T2, 35\% $(n=107)$ reported experiencing at least one "new" trauma; all but one also reported a lifetime trauma at T1. Of these recent events, "other" events were most commonly nominated as the worst ( $n=48$, e.g., Elementary School Shootings, Boston Bombings, Hurricane Sandy). Of participants displaying elevated ( $>44)$ PTSD symptoms at T1 ( $n=214$, of whom 78 also completed T2), 56.4\% $(n=44)$ showed persistently elevated PTSD symptoms at T2, and $43.6 \%(n=34)$ were classified as recovered.

Table 1 also shows the cross-sectional relationships between the demographic, cognitive, metacognitive and symptom variables at T1 and T2. Females reported significantly more PTSD symptoms than males at T1, $t(662)=-2.84, p<.01, d=.24$. Age at trauma was significantly and negatively related to PTSD. Number of prior traumas, emotional distress at the time of the trauma, anxiety, depression, and all types of cognition and metacognition were positively and significantly associated with PTSD symptoms at both time points. Participants' negative cognitions about the self were highly correlated with other variables; in particular with PTSD $(r=.70)$ and RIQ $(r=.83)$. Thus, we excluded this variable from our regression analyses due to multicollinearity. ${ }^{3}$

\footnotetext{
${ }^{2}$ Table S1 in the Supplemental Material available online shows the lifetime prevalence of each potentially traumatic event, as well as participants' exposure to these events between T1 and T2.

${ }^{3}$ We tested our hypotheses using ordinary least squares (OLS) regression and logistic regression analyses. One might be concerned that the predictors in our models could be highly correlated. To address this concern, we verified each of our analyses using ridge regression (Hoerl \& Kennard, 1970; Maydeu-Olivares \& Millsap, 2009). Ridge regression improves a model's predictive accuracy in cases where there are more predictors than observations, and/or when the predictors are highly correlated. The ridge regression results did not differ from
} 
We next conducted a (forced entry) hierarchical regression to examine whether certain demographic, cognitive and metacognitive variables predicted the degree of concurrent PTSD symptomatology at $\mathrm{T}^{4}$. In Step 1, we entered five 'control' variables-age at trauma, distress at the time of recent worst trauma exposure, gender, number of prior traumas, and depression; these variables accounted for a significant proportion of the variance in symptoms (43\%). All were independent predictors except gender and distress; depression, which is often comorbid with PTSD (APA, 2013), was the strongest predictor. In Step 2, pre-existing cognitive beliefs accounted for a significant additional proportion of symptom variance $(10 \%)$; depression, age at trauma, and number of prior traumas remained significant predictors, and world beliefs and self-blame were also independent predictors. In Step 3, metacognitive beliefs also accounted for a significant proportion of symptom variance over and above the control and cognitive variables (11\%). Depression, prior traumas, and world beliefs from Step 2 remained significant predictors, along with beliefs concerning the uncontrollability and danger of thoughts, positive and negative meta-memory beliefs, and negative inferences about intrusive memories. The overall model explained $64 \%$ total variance in PTSD, $F(13,348)=47.96, p<.001$.

We next examined whether pre-existing metacognitive beliefs predicted degree of PTSD symptomatology following recent trauma exposure (i.e., exposure between T1 \& T2), we conducted a (forced entry) hierarchical regression. Here, we included only participants who had experienced a novel trauma $(n=107)$ between T1 and T2, with T2 PCL as the outcome variable. Of these, 25 participants had no score for the RIQ, because it was only administered to participants who reported intrusions. To maximize the available sample size, we used the Expectation-Maximization (EM) technique in SPSS to replace missing values

those obtained with OLS and logistic regression; hence, we report only the latter ones for ease of interpretation.

${ }^{4}$ Table S2 in the Supplemental Material available online displays the full regression table. 
for participants who did not report age at the time of the trauma $(n=3)$, distress relating to the trauma $(n=7)$, or either of these variables $(n=2)$. We entered six control variables - age and distress at the time of recent trauma exposure, depression, gender, number of prior traumas, and whether or not the person had elevated PTSD symptom levels at T1-simultaneously in Step 1. We added the cognitive variables (T1) in Step 2 and the metacognitive variables (T1) in Step 3.

Table 2 displays the regression statistics for each step of the model. As shown in Step 1, the control variables accounted for a significant proportion of variance in T2 PTSD symptoms (55\%). In Step 2, pre-existing cognitive beliefs did not explain significant additional variance (1\%). However, as predicted, in Step 3 pre-existing metacognitive beliefs did account for a significant proportion of additional variance (13\%). In particular, preexisting beliefs concerning the uncontrollability/danger of thoughts and negative inferences about intrusive memories significantly and independently predicted PTSD symptoms after recent trauma. These variables are important contributors to the final model, uniquely accounting for $5.29 \%$ and $2.96 \%$ of the variance respectively. Other critical variables are depression (5.95\%) and prior traumas (3.39\%). However, contrary to our hypothesis, people who, at T1, did not strongly believe that they needed to control their thoughts had more symptoms of PTSD at T2 (2.46\%). The overall model explained $69 \%$ total variance in PTSD, $F(14,67)=10.47, p<.001$.

We also predicted that maladaptive meta/cognitions would drive the persistence of PTSD and hence differentiate people who experienced elevated PTSD symptoms between T1 and T2 and people whose symptoms abated. Using only those participants whose symptoms were classified as either 'persistent' or 'recovered' at T2 (n=78, less 12 participants with no RIQ score), we entered the cognitive and metacognitive variables into a (forced entry) 
hierarchical multivariate logistic regression analysis. ${ }^{5}$ This model accurately predicted $83.3 \%$ of cases and contained only negative meta-memory beliefs ( $\mathrm{OR}=1.35[1.01,1.81], p<.05)$, and negative inferences about the meaning of intrusions $(\mathrm{OR}=1.17[1.03,1.33], p=.01)$ as significant predictors of persistent PTSD symptoms $\left(\chi^{2}=38.61, p<.01\right)$.

\section{Discussion}

We examined the relationship between specific types of metacognition and the development and maintenance of posttraumatic stress symptoms. Considering the correlational data, at $\mathrm{T} 1$ and $\mathrm{T} 2$, the more our participants held unhelpful beliefs, the more PTSD symptomatology they showed. Interestingly, cognition about the self was the most important correlate of PTSD symptomatology, corroborating previous research (vs. world/self-blame; Bryant \& Guthrie, 2005; Foa \& Rauch, 2004; Moser, Hajcak, Simons, \& Foa, 2007). This result is not surprising, given substantial conceptual overlap between negative self-related cognition, and symptoms, as well as with our other key variables. Of the metacognitive beliefs, at $\mathrm{T} 1$ negative meta-memory beliefs were the most important while at T2 it was beliefs about intrusions. However, these particular results do not speak to whether pre-trauma metacognition predicts later PTSD symptomatology.

Thus, we examined whether cognitive and metacognitive factors predicted PTSD symptomatology after trauma exposure. We found that pre-existing cognitive and metacognitive beliefs (T1) predicted PTSD symptom levels after exposure to a novel trauma (T2). Specifically, people reported more PTSD symptoms when exposed to trauma if they had showed concern that their thoughts were dangerous, and made negative inferences about the meaning of their intrusive symptoms. In addition, people who, pre-trauma, believed the

\footnotetext{
${ }^{5}$ Due to the reduced sample size available for analysis, we included only the theoretical predictors of interest (i.e., cognitive and metacognitive factors) here. Table S3 in the Supplemental Material available online displays the full regression table.
} 
world to be more safe and predictable, and did not believe that they should control their thoughts, were also more likely to report more symptoms post-trauma. Perhaps for people with overly-optimistic views of the safety and fairness of the world, trauma shatters their basic beliefs and leads them to shift those beliefs to the other extreme (Janoff-Bulman, 1992; Foa \& Riggs, 1993). Our data, like Bryant and Guthrie's (2005), suggest beliefs that the world is dangerous and that it is bad to think certain thoughts, are activated post — rather than pretrauma exposure (c.f., Foa \& Riggs, 1993). Finally, we found the extent to which people held meta-memory beliefs that fragmentary trauma memory was pathological, and made negative inferences about the meaning of intrusions played a significant role in maintaining elevated PTSD symptoms over twelve-weeks. These results support the hypothesis that metacognition plays an important role in PTSD maintenance among adults.

Taken together, our results support the metacognitive model of PTSD (Wells, 2000): Problematic metacognitions likely blocked participants' adaptation process, increasing their focus on threat such that anxiety and a sense of ongoing danger persisted, which in turn maintained symptoms (e.g., Roussis \& Wells, 2006). Our data also contribute to a growing literature showing the importance of meta-memory beliefs (about intrusive memories and memory deficits) to PTSD symptoms. In particular, negative beliefs that a fragmented memory means something bad, or is abnormal, predicted PTSD maintenance.

Whether people with PTSD have fragmentary and disorganized memories for their traumatic experience has long been debated (e.g., Ehlers, Ehring, \& Kleim, 2012). Our data suggest that negative metacognition about traumatic memory might contribute to traumaexposed people's struggle to resolve their symptoms. Interestingly, people's metacognition becomes more apparent the more they report recurrently thinking about an event. The more we think about an event, the more we might think details are missing from memory. Recovering previously inaccessible details creates an impression of partial amnesia (e.g., 
Bernsten \& Rubin, 2014). Yet, non-clinical research suggests this is not unique to traumatic memory (Read \& Lindsay, 2000). Thus, it is unclear whether disorganization in traumatic memories contributes significantly to PTSD symptoms, or if meta-memory beliefs and strategies concerning trauma memory (e.g., rumination, 'gap filling') play a greater role in maintaining symptoms. We do know, however, that rumination mediates the relationship between meta-memory beliefs and intrusive PTSD symptoms (Bennett \& Wells, 2010).

There are several limitations to our study. First, although we gathered data prospectively, the study lasted only twelve-weeks. In addition, because not all participants met the one-month duration criterion for PTSD with symptoms at T2, it is possible that, amongst this group, symptomatology represented normal stress reactions to trauma. Importantly, we did not include a clinical diagnostic interview to assess PTSD or any other psychopathology at $\mathrm{T} 1$ that could potentially account for our results. Also, we used a convenience sample of students and a broad section of internet users, who were predominantly Caucasians from the USA, which may limit the generalizability of our findings. Moreover, because the participants reporting the highest levels of problematic metacognition and PTSD symptoms at T1 were less likely to complete the entire study, our final T2 sample was not representative of those at the more extreme end. Also, although we initially recruited a large sample, due to incomplete data on some measures, the final sample size we used for our main regression analyses was limited. Finally, in interpreting the separate contribution of metacognitions to the development and maintenance of posttraumatic stress symptoms, it is important to acknowledge that the RIQ could potentially also be indexing cognitions, rather than just pure meta-cognitions.

Considering our key findings, and the limitations of our study, we would suggest two future research areas. To advance our understanding of how metacognitive beliefs may lead to PTSD after new trauma exposure, we suggest conducting a similar longitudinal study with 
people at high risk of trauma exposure, such as emergency service personnel (e.g., Bryant \& Guthrie, 2007). Research could also examine whether cognitive bias training (Woud et al., 2012) directed specifically at changing problematic metacognitive beliefs halts the development of symptoms after new trauma, or reduces symptoms relating to previous trauma. This method would help determine whether metacognitive beliefs are causal risk factors for PTSD (Kraemer, Kazdin, Offord, Kessler, Jensen \& Kupfer, 1997). In both lines of research, we suspect it will be important to employ a longer delay when assessing the role of metacognition in maintaining PTSD symptoms over time, and to attempt to capture metacognition and symptoms immediately after trauma exposure.

In summary, our results indicate that metacognition-particularly regarding traumatic memory, a new area of investigation - plays an active role in adult PTSD. Specifically, this evidence adds to growing empirical support that metacognitive beliefs are important to predicting PTSD symptomatology. 


\section{References}

American Psychiatric Association. (2013). Diagnostic and statistical manual of mental disorders. (5th ed.). Washington, DC: Author.

Belsley, D. A., Kuh, E., \& Welsch, R. E. (2005). Regression diagnostics: Identifying influential data and sources of collinearity. New York: John Wiley \& Sons, Inc.

Bennett, H., \& Wells, A. (2010). Metacognition, memory disorganisation and rumination in posttraumatic stress symptoms. Journal of Anxiety Disorders, 24, 318-325. DOI: 10.1016/j.janxdis.2010.01.004

Berntsen, D. \& Rubin, D.C. (2014). Involuntary memories and dissociative amnesia: Assessing key assumptions in PTSD research. Clinical Psychological Science, 2, 174 186. DOI: $10.1177 / 2167702613496241$.

Blanchard, E. B., Jones-Alexander, J., Buckley, T.C., \& Forneris, C. A. (1996). Psychometric properties of the PTSD Checklist (PCL). Behaviour Research and Therapy, 34, 669673. DOI: $10.1016 / 0005-7967(96) 00033-2$

Bonanno, G. A. (2005). Resilience in the face of potential trauma. Current Directions in Psychological Science, 14, 135-148. DOI: 10.1111/j.0963-7214.2005.00347.x

Breslau, N., Kessler, R. C., Chilcoat, H. D., Schultz, L. R., Davis, G. C., \& Andreski, P. (1998). Trauma and posttraumatic stress disorder in the community: The 1996 Detroit area survey of trauma. Archives of General Psychiatry, 55, 626-632. DOI: 10.1001/archpsyc.55.7.626

Bryant, R. A., \& Guthrie, R. M. (2005). Maladaptive appraisals as a risk factor for posttraumatic stress: A study of trainee firefighters. Journal of Psychological Science, 16, 749-752. DOI: 10.1111/j.1467-9280.2005.01608.x

Carlson, E. B., Smith, S. R., Palmieri, P. A., Dalenberg, C., Ruzek, J. I., Kimerling, R., Burling, T. A., \& Spain, D. A. (2011). Development and validation of a brief self- 
report measure of trauma exposure: the Trauma History Screen. Psychological Assessment, 23, 463-477. DOI: doi.org/10.1037/a0022294

Clohessy, S., \& Ehlers, A. (1999). PTSD symptoms, response to intrusive memories and coping in ambulance service workers. British Journal of Clinical Psychology, 38, 251-265. DOI: 10.1348/014466599162836

Ehlers, A., Ehring, T., \& Kleim, B. (2012). Information processing in Posttraumatic Stress Disorder. In J. G. Beck \& D. M. Sloan (Eds.), The Oxford Handbook of Traumatic Stress Disorders (pp. 191-218). Oxford: Oxford University Press.

Ehlers, A., Mayou, R. A., and Bryant, B. (1998). Psychological predictors of posttraumatic stress disorder after motor vehicle accidents. Journal of Abnormal Psychology, 107, 508-519. DOI: 10.1037//0021-843X.107.3.508

Foa, E. B., Ehlers, A., Clark, D. M., Tolin, D. F., \& Orsillo, S. M. (1999). The posttraumatic cognitions inventory (PTCI): development and validation. Journal of Psychological Assessment, 11, 303-314. DOI: 10.1037/1040-3590.11.3.303

Foa, E. B., \& Rauch, S. M. (2004). Cognitive changes during prolonged exposure versus prolonged exposure plus cognitive restructuring in female assault survivors with Posttraumatic Stress Disorder. Journal of Consulting and Clinical Psychology, 72, 879-884. DOI: 10.1037/0022-006X.72.5.879

Foa, E. B., \& Riggs, D. S. (1993). Post-traumatic stress disorder in rape victims. In J. Oldham, M. B. Riba, \& A.Tasman, Annual review of psychiatry (Vol. 12) (pp. 273303). Washington, DC: American Psychiatric Association.

Frazier, P., Anders, S., Perera, S., Tomich, P., Tennen, H., Park, C., \& Tashiro, T. (2009). Traumatic events among undergraduate students: prevalence and associated symptoms. Journal of Counselling Psychology, 56, 450-460. DOI: 10.1037/a0016412 
Halligan, S. L., Michael, T., Clark, L. M., \& Ehlers, A. (2003). Posttraumatic stress disorder following assault: the role of cognitive processing, trauma memory, and appraisals. Journal of Consulting and Clinical Psychology, 71, 419-431. DOI: 10.1037/0022006X.71.3.419

Hoerl, A. E., \& Kennard, R. W. (1970). Ridge regression: Biased estimation for nonorthogonal problems. Technometrics, 12, 55-67. DOI: $10.1080 / 00401706.1970 .10488634$

Janoff-Bulman, R. (1992). Shattered assumptions: toward a new psychology of trauma. New York: The Free Press.

Kleim, B., Grey, N., Wild, J.,; Nussbeck, F. W., Stott, R., Hackmann, A., Clark, D. M., \& Ehlers, A. (2013). Cognitive change predicts symptom reduction with cognitive therapy for posttraumatic stress disorder. Journal of Consulting and Clinical Psychology, 81, 383-393. DOI: 10.1037/a0031290

Kraemer, H.C., Kazdin, A. E., Offord, D. R., Kessler, R.C., Jensen, P. S., \& Kupfer, D. J. (1997). Coming to terms with the terms of risk. Archives of General Psychiatry, 54, 337-343. DOI: 10.1001/archpsyc.1997.01830160065009

Lee, D. (2006). Case conceptualisation in complex PTSD: Integrating theory with practice. In D. H. Barlow (Ed.), Clinical handbook of psychological disorders: A step by step treatment manual (pp.188-215). London: The Guilford Press.

Meiser-Stedman R., Dalgleish T., Glucksman E., Yule W., \& Smith P. (2009). Maladaptive cognitive appraisals mediate the evolution of posttraumatic stress reactions: A 6month follow-up of child and adolescent assault and motor vehicle accident survivors. Journal of Abnormal Psychology, 118, 778-87. DOI: 10.1037/A0016945.

Millsap, R. E., \& Maydeu-Olivares, A. (Eds.) (2009). The SAGE Handbook of Quantitative Methods in Psychology. London: SAGE Publications, Ltd. 
Moser, J. S., Hajcak, G., Simons, R. F., \& Foa, E. B. (2007). PTSD symptoms in traumaexposed college students: The role of negative cognitions, trauma type, and anxiety. Journal of Anxiety Disorders, 21, 1039-1049. DOI: 10.1016/j.janxdis.2006.10.009.

Read, J. D., \& Lindsay, D. S. (2000). “Amnesia” for summer camps and high school graduation: memory work increases reports of prior periods of remembering less. Journal of Traumatic Stress, 13, 129-147. DOI: 10.1023/A:1007781100204

Robinaugh, D. J., Marques, L., Traeger, L. N., Marks, E. H., Sung, S. C., Gayle, Beck, J. G., Pollack, M. H., \& Simon, N. M. (2011). Understanding the relationship of perceived social support to post-trauma cognitions and posttraumatic stress disorder. Journal of Anxiety Disorders, 25(8), 1072-1078. DOI: 10.1016/j.janxdis.2011.07.004

Roussis, P., \& Wells, A. (2006). Posttraumatic stress symptoms: Tests of relationships with thought control strategies and beliefs as predicted by the metacognitive model. Personality and Individual Differences, 40, 111-112. DOI:10.1016/j.paid.2005.06.019

Weathers, F. W., Litz, B. T., Herman, D. S., Huska, J. A., \& Keane, T. M. (1993). The PTSD Checklist: Reliability, validity, and diagnostic utility. Paper presented at the annual meeting of the International Society for Traumatic Stress Studies, San Antonio, TX.

Wells, A. (2000). Emotional disorders and metacognition: innovative cognitive therapy. Chichester, UK: Wiley.

Wells, A. \& Cartwright-Hatton, S. (2004). A short form of the metacognitions questionnaire: properties of the MCQ-30. Behaviour Research Therapy, 42, 385-96. DOI: 10.1016/S0005-7967(03)00147-5

Wells, A., \& Sembi, S. (2004). Metacognitive therapy for PTSD: A preliminary investigation of a new brief treatment. Journal of Behaviour Therapy and Experimental Psychiatry, 35, 307-318. DOI: 10.1016/j.jbtep.2004.07.001 
Woud, M. L., Holmes, E. A., Postma, P., Dalgleish, T., \& Mackintosh, B. (2012). Ameliorating intrusive memories of distressing experiences using computerized reappraisal training. Emotion, 12, 778-784. DOI: 10.1037/a0024992

Zigmund, A. S., \& Snaith, R. P. (1983). The hospital anxiety and depression scale. Acta Psychiatrica Scandinavica, 67, 361-370. DOI: 10.1111/j.1600-0447.1983.tb09716.x 
Authors' Contribution

M.T. and D.S. developed the study concept. All authors contributed to the study design. R.S. performed data collection; R. S. performed initial analyses and interpretation; M. T. performed additional analysis. R. S. and M. T. drafted the paper, and D.S. and H.F. provided critical revisions. All authors approved the final version of the paper for submission. 
Acknowledgements

RS was supported by an award from the NAHSSS funded by the Commonwealth Department of Health and Ageing. ${ }^{6}$ This planning phase of this research was supported by a Wellcome Trust Biomedical Vacation Scholarship supervised by MT and HF. We thank Caitlin Lloyd, Evan Dawson and Hannah James for their assistance in preparing study materials, Jacinta Oulton and Ella Moeck for their assistance with data preparation, and James Rockey for his advice on ridge regression.

\footnotetext{
${ }^{6}$ The views expressed in this research do not necessarily represent those of the NAHSSS, its Administrator, Services for Australian Rural and Remote Allied Health (SARRAH) and/or the Government Department of Health and Ageing.
} 
Table 1

Demographic, Trauma-Related Characteristics, and Main Measures for the Total Sample including Cross-

Sectional Pearson's Correlation Coefficients and Confidence Intervals with PTSD Symptoms.

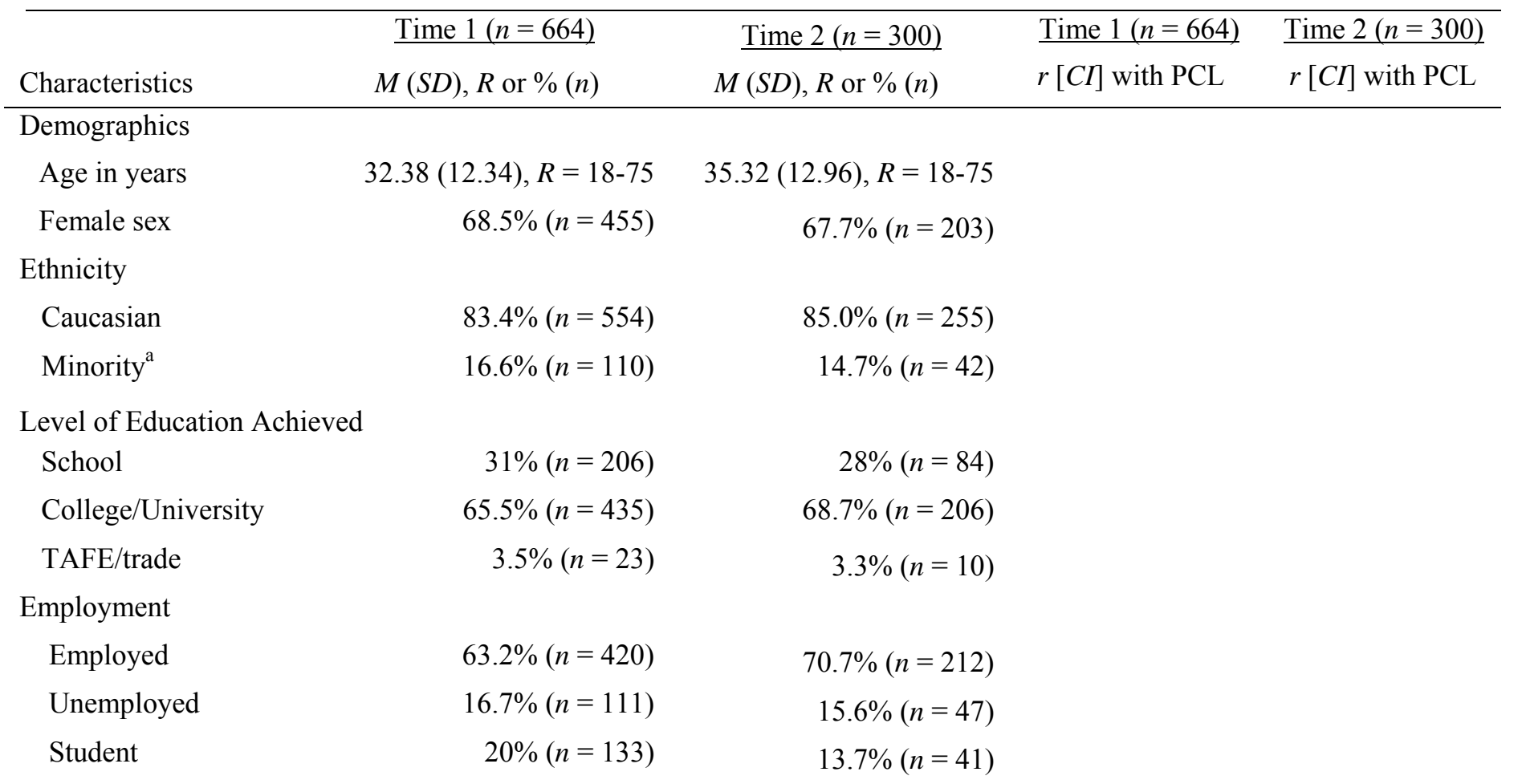

Trauma Characteristics and Symptoms

\begin{tabular}{|c|c|c|c|c|}
\hline Age at trauma & $20.80(11.56), R=0-70$ & $35.32(12.96), R=18-75$ & $-.19[-.27,-.11]^{* * *}$ & $-.18[-.29, .07]^{* *}$ \\
\hline Time since trauma ${ }^{b}$ & $12.12(11.44), R=0-57$ & $26.04(22.02), R=0-105$ & $-.08[-.16,-.01]$ & $.05[-.15,26]$ \\
\hline Number of traumas & $4.06(2.72), R=0-13$ & $4.36(2.99), R=0-15$ & $.38[.31, .44]^{* * *}$ & $.41[.32, .51]^{* * *}$ \\
\hline Distress at trauma & $4.59(.79), R=1-5$ & $3.93(1.38), R=1-5$ & $.21[.14, .28]^{* * *}$ & $.43[.26, .58]^{* * *}$ \\
\hline HADS total & $16.67(7.06), R=0-42$ & $12.80(8.29), R=0-42$ & $.68[.64, .72]^{* * *}$ & $.74[.70, .77]^{* * *}$ \\
\hline HADS anxiety & $10.40(4.25), R=0-21$ & $7.61(4.62), R=0-21$ & $.64[.59, .70]^{* * *}$ & $.70[.66, .74]^{* * *}$ \\
\hline HADS depression & $6.27(3.63), R=0-21$ & $5.18(4.52), R=0-21$ & $.56[.49, .62]^{* * *}$ & $.64[.59, .68]^{* * *}$ \\
\hline PCL total & $37.09(17.38), R=17-85$ & $32.52(14.97), R=17-84$ & - & - \\
\hline \multicolumn{5}{|l|}{ Metacognition Measures } \\
\hline BAMQ positive & $14.38(6.27), R=8-32$ & $15.34(6.75), R=9-36$ & $.44[.38, .50]^{* * *}$ & $.56[.48, .63]^{* * *}$ \\
\hline BAMQ negative & $10.01(3.98), R=7-28$ & $10.72(3.98), R=8-32$ & $.58[.52, .62]^{* * *}$ & $.60[.52, .67]^{* * *}$ \\
\hline MCQ positive worry & $10.84(4.35), R=6-24$ & $10.60(4.10), R=6-24$ & $.24[.17, .31]^{* * *}$ & $.30[.19, .40]^{* * *}$ \\
\hline MCQ uncontrollability & $13.2(5.17), R=6-24$ & $12.29(4.87), R=6-24$ & $.54[.48, .59]^{* * *}$ & $.53[.44, .61]^{* * *}$ \\
\hline MCQ thought control & $11.84(4.03), R=6-24$ & $11.04(4.14), R=6-24$ & $.43[.37, .49]^{* * *}$ & $.39[.29, .48]^{* * *}$ \\
\hline PTCI self & $2.65(1.42)=1-7$ & $2.46(1.37), R=1-7$ & $.71[.67, .74]^{* * *}$ & $.76[.71, .80]^{* * *}$ \\
\hline PTCI world & $4.23(1.65), R=1-7$ & $3.92(1.69), R=1-7$ & $.57[.51, .61]^{* * *}$ & $.56[.48, .63]^{* * *}$ \\
\hline PTCI self-blame & $2.53(1.58), R=1-7$ & $2.32(1.56), R=1-7$ & $.46[.40, .52]^{* * *}$ & $.43[.33, .52]^{* * *}$ \\
\hline RIQ intrusions & $14.81(9.36), R=6-42$ & $13.86(8.79), R=6-42$ & $.70[.64, .74]^{* * *}$ & $.76[.69, .81]^{* * *}$ \\
\hline
\end{tabular}

Note. ${ }^{*} p<.05 .{ }^{* *} p<.01 .{ }^{* *} p<.001{ }^{\mathrm{a}}$ Minority, e.g., African American, Hispanic, Middle Eastern, Asian American, multiracial.

There were no significant differences in PTSD symptoms between white and non-white participants at either time point, and we therefore excluded ethnicity from further analysis. ${ }^{\mathrm{b}}$ Time since trauma $=$ years at $\mathrm{T} 1$, days at $\mathrm{T} 2$. 
Table 2

Summary of Hierarchical Regression of Control Variables, Cognitive Beliefs and Metacognitive Beliefs at Time 1 in Predicting Posttraumatic Stress Symptoms at Time 2

\begin{tabular}{|c|c|c|c|c|c|}
\hline Predictor & B & SE & $\beta$ & $\begin{array}{c}\text { Squared } \\
\text { semi-partial } \\
\text { correlation }\end{array}$ & $\overline{\mathrm{VIF}}$ \\
\hline \multicolumn{6}{|l|}{ Step 1} \\
\hline Constant & -1.99 & 7.82 & & & \\
\hline Age at time of trauma & -.04 & .10 & -.03 & .001 & 1.04 \\
\hline Number of traumas & .38 & .46 & .06 & .004 & 1.19 \\
\hline Distress at trauma & 3.69 & 1.59 & $.19^{*}$ & .030 & 1.25 \\
\hline Gender & -.82 & 3.13 & -.02 & $<.001$ & 1.29 \\
\hline Time 1 PTSD & .43 & .10 & $.45^{* * *}$ & .111 & 1.82 \\
\hline Time 1 Depression & 1.44 & .47 & $.30 * *$ & .052 & 1.75 \\
\hline \multicolumn{6}{|c|}{$R^{2}=.58, F(6,75)=17.53, p<.001$} \\
\hline \multicolumn{6}{|l|}{ Step 2} \\
\hline Constant & 3.52 & 8.70 & & & \\
\hline Age at time of trauma & -.04 & .10 & -.03 & .001 & 1.05 \\
\hline Number of traumas & .54 & .47 & .10 & .007 & 1.25 \\
\hline Distress at trauma & 3.07 & 1.65 & .16 & .019 & 1.35 \\
\hline Gender & -.23 & 3.14 & -.01 & $<.001$ & 1.31 \\
\hline Time 1 PTSD & .49 & .11 & $.52 * * *$ & .112 & 2.39 \\
\hline Time 1 Depression & 1.68 & .49 & $.35^{* *}$ & .064 & 1.94 \\
\hline PTCI world & -1.89 & 1.23 & -.16 & .013 & 1.97 \\
\hline PTCI self-blame & -.09 & .97 & -.01 & $<.001$ & 1.68 \\
\hline \multicolumn{6}{|c|}{$R_{\text {Change }}^{2}=.01, \mathrm{~F}_{\text {Change }}(2,73)=1.26, p=.289$} \\
\hline \multicolumn{6}{|l|}{ Step 3} \\
\hline Constant & 3.51 & 8.91 & & & \\
\hline Age at time of trauma & -.00 & .10 & -.00 & $<.001$ & 1.27 \\
\hline Number of traumas & 1.03 & .44 & $.18^{*}$ & .025 & 1.34 \\
\hline Distress at trauma & 2.02 & 1.62 & .11 & .007 & 1.56 \\
\hline Gender & .40 & 3.15 & .01 & $<.001$ & 1.59 \\
\hline Time 1 PTSD & .25 & .12 & $.26^{*}$ & .020 & 3.37 \\
\hline Time 1 Depression & 1.46 & .49 & $.31 * *$ & .041 & 2.30 \\
\hline PTCI world & -2.89 & 1.17 & $-.25 *$ & .028 & 2.14 \\
\hline PTCI self-blame & -.40 & .94 & -.04 & .001 & 1.89 \\
\hline RIQ intrusions & .44 & .17 & $.28 *$ & .032 & 2.36 \\
\hline BAMQ positive meta-memory & .20 & .22 & .08 & .004 & 1.68 \\
\hline BAMQ negative meta-memory & .14 & .38 & .04 & .001 & 2.39 \\
\hline MCQ positive worry & .15 & .30 & .04 & .001 & 1.36 \\
\hline MCQ uncontrollability/danger & 1.04 & .36 & $.31 * *$ & .038 & 2.52 \\
\hline MCQ control thoughts & -.84 & .41 & $-.22 *$ & .020 & 2.48 \\
\hline
\end{tabular}

Note. ${ }^{*} p<.05 .{ }^{* *} p<.01 .{ }^{* * *} p<.001$. 
